\title{
Combining post-specimen aberration correction and direct electron detection to image molecular structure in liquid crystal polymers
}

\author{
Eric. A. Stach, ${ }^{1}$ Jing Li, ${ }^{1}$ Huolin Xin, ${ }^{1}$ Dmitri Zakharov, ${ }^{1}$ Yo Hon Kwon, ${ }^{2}$ and Elsa Reichmanis ${ }^{2}$ \\ 1. Center for Functional Nanomaterials, Brookhaven National Laboratory, Upton, NY 11973 \\ 2. School of Chemical and Biomolecular Engineering, Georgia Institute of Technology, Atlanta, GA
}

Transmission electron microscopy studies of polymeric materials are greatly compromised by the extreme radiation sensitivity that these materials display. In particular, this has greatly inhibited the applicability of high-resolution phase contrast imaging (HR-TEM) approaches for exploring the structure of (semi-)crystalline polymeric materials. This is because HR-TEM generally requires substantial electron doses to obtain the requisite signal to noise in the image. However, with the recent advent of direct electron detector technologies [1], the time is ripe to revisit the question as to whether or not HR-TEM methods can be applied to these important materials systems.

In this work, we demonstrate how the combination of aberration-corrected phase contrast electron microscopy, direct electron detection and low dose methods can be used to provide molecular scale (atomic) resolution images of the structure of an important liquid crystalline polymer, poly-(3hexylthiopene) (P3HT). In particular, we take advantage of the high detection quantum efficiency of a Gatan K2-IS Direct Electron Detector [2], operating in the "counting mode", a low dose mode wherein every primary electron that that impinges on the detector is located, and both the read-out noise and stochastic distribution of energy deposited in traditional scintillator-based systems are eliminated as sources of noise. This allows an effective doubling of the detectable information beyond the traditional Nyquist frequency limit [3]. Utilization of the counting mode allows the acquisition of a series of very low dose images (of order 1-10 electrons $/ \AA^{\wedge} 2$ ) to be acquired sequentially, and cross-correlated to obtain an image with maximum signal to noise. Importantly, the serial acquisition allows identification of the maximum dose that the material can withstand, as image degradation is directly visible in the series.

In this presentation, we will focus on issues associated with appropriate image acquisition parameters for HR-TEM imaging of P3HT, associated di-block copolymers made of P3HT/ Polydimethylsiloxane (PDMS), as well as other liquid crystalline polymeric systems. Insights regarding the ability to image polymeric chains with these structures, as well as th4 "tie chains" that have been proposed to provide charge transport pathways between nanoaggregated structures will be presented. We will also describe how we are automating the image acquisition process, through the use of the UCSF Image [4] and SerialEM [5] software packages.

\section{References:}

[1] Battaglia M, Contarato D, Denes P, Doering D, Giubilato P, Kim T S, Mattiazzo S, Radmilovic V and Zalusky S, Nucl Instrum Meth A 598 2009, pp. 642-9

[2] http://www.gatan.com/products/tem-imaging-spectroscopy/k2-camera

[3] McMullan G, Clark A T, Turchetta R and Faruqi A R 2009 Enhanced imaging in low dose electron microscopy using electron counting Ultramicroscopy 109, 2009, pp. 1411-6

[4] http://cryoem.ucsf.edu/software/UCSFImage.html

[5] http://bio3d.colorado.edu/SerialEM/ 
[6] This work was supported as part of the Center for Mesoscale Transport Properties, an Energy Frontier Research Center funded by the U.S. Department of Energy, Office of Science, Basic Energy Sciences under Award \# DE-SC0012673. This research used resources of the Center for Functional Nanomaterials, which is a U.S. DOE Office of Science Facility, at Brookhaven National Laboratory under Contract No. DE-SC0012704

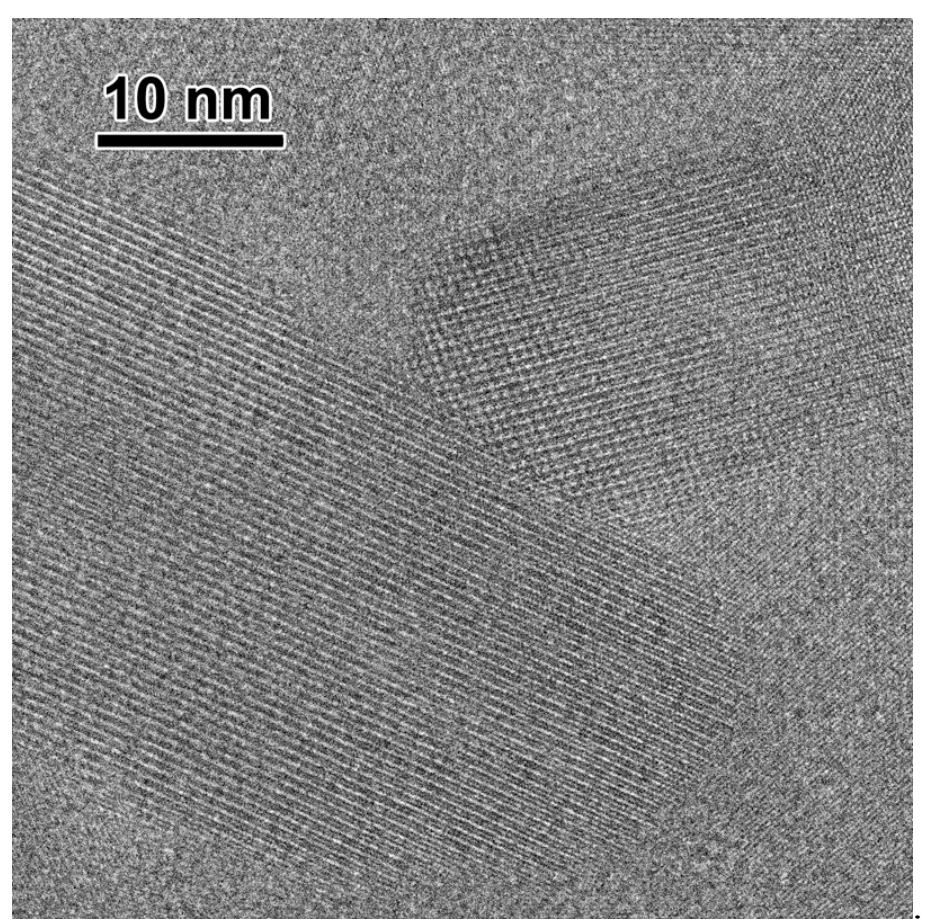

Figure 1. High-resolution phase contrast image of the junction between two P3HT crystalline domains. Aligned summation of 20 images, each acquired at $12.5 \mathrm{e}^{-} / \AA^{2}$

Figure 2. High-resolution phase contrast image of a well-aligned P3HT domain. Aligned summation of 20 images, each acquired at $12.5 \mathrm{e}^{-} / \AA^{2}$.

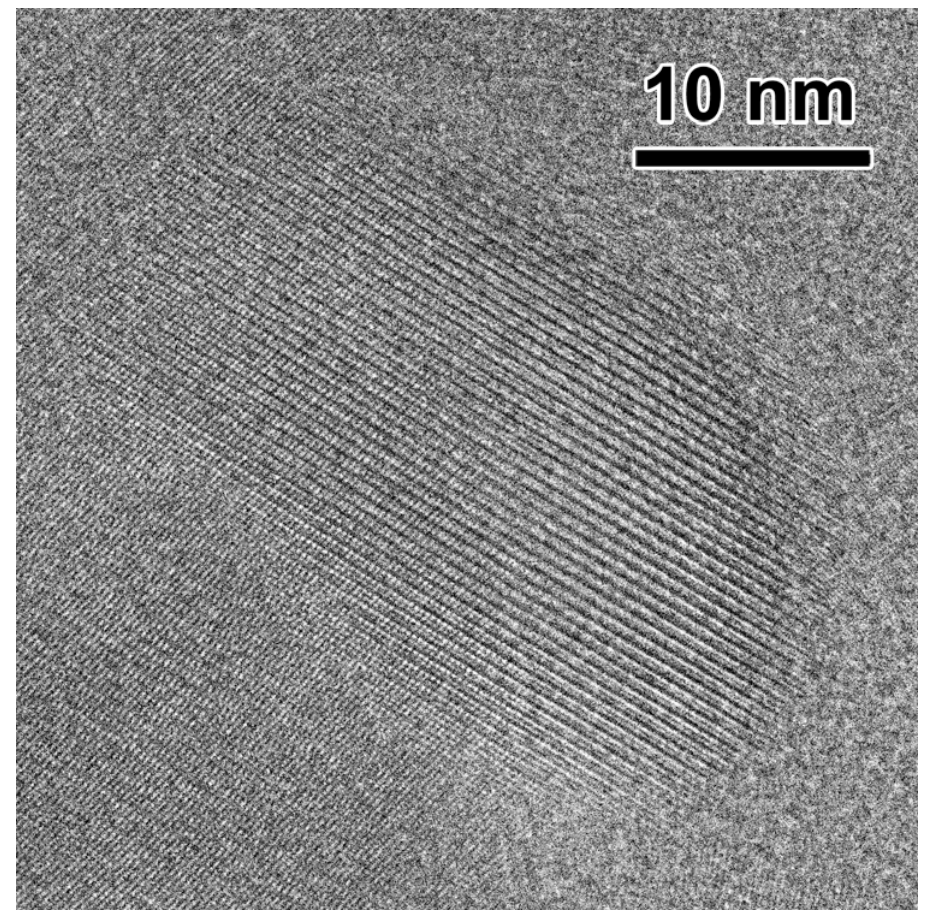

